Theories \& Applications, the International Edition

Printed Version: (ISSN 2090-5262)

Online Version: (ISSN 2090-5270)

November 2013, Volume 3, No. 3 Pages (78 - 86)

\title{
An Electronic Device for Identifying the Ultimate Gear Ratio According to Pedal Rotation Rate and Bike Velocity
}

\author{
Mohamed GaberBrekaa*, Ali Mohamed Abd El-Rahman Al-Zahaby**, Ebraheem Mohamed \\ Farouk Gabr***, Yaser Ali Mahmoud Abd El-Rahman***
}

\section{Background and Problem}

$\mathrm{T}$ The world is currently witnessing major developments in sports achievements due to continuous efforts of scientists through the interaction of physical education sciences with other fields of knowledge to improve the sports achievement levels. Nowadays, sports achievements and world records are being established and broken before even getting involved in sports competitions. This is due to scientific research and studies. Therefore, matches and competitions are now being prepared in labs.

It is agreed that there are individual differences among persons. These differences lead, naturally, to differences in individual performance of motor skills. During the past few decades, sports motion witnessed a giant leap that made the limits of human ability to exceed all barriers and to improve through establishing records that were once mere fantasy. This great improvement in sports is not merely a coincidence. Instead, it is the fruit of scientific and field efforts in such sports through the results of scientific research in other disciplines that provide sports with all that needs to improve.

Kinesiology is the science of human motor performance. It studies the human motion. In the sports field, kinematics is trying to study the

\footnotetext{
* Professor of Bio-Mechanics, Head of Kinesiology Dept, Faculty of Physical Education - Tanta University - Egypt

** Professor of Mechanical Engineering, Head of Power Mechanics Dept, Faculty of Engineering - Tanta University Egypt

*** Assistant Professor, Kinesiology Dept, Faculty of Physical Education - Tanta University - Egypt

**** Lecturer, Physical Education Dept, The Higher Institute for Technology-10th Ramadan City - Egypt
}

sports motion and its components to improve and correct the sports technique through modern training approaches. The successful coach is the one who is fully aware of nature of motion, its components and its governing scientific principles.

During the past few years, motor learning and sports training are greatly affected by the technological revolution. The training process has taken its form, structure and organization according to modern developments in approaches and equipments used in it. Scientific analysis of the physical education field undeniably indicates great developments in sports training and motor learning.

There are various equipments for the training process that help improving the technical performance levels of athletes. These equipments develop power, strength, velocity and endurance as it was designed not to deteriorate the technical performance of athletes. Training with technical equipments has major effects on improving athletes' digital records (1).

Sports engineering is a new branch of human engineering that links and coordinates physical education, sports and various branches of engineering. Major universities all over the world paid more attention to this new branch. This is clear in the fruitful cooperation in De Mont Fort University, through Leister College and Bedford College for Humanities and Physical Education, Sports Engineering Technology, innovations and designs on the bases of industrial and sports production. These technologies are linked to sports and physical education sciences, especially measurement and evaluation and the study and evaluation of human body and its structure. It is also related 
to the study of motor performance mechanics and its relation with tools, equipments and facilities in general and sports equipments specifically (2).

David Swain (1998) indicated that cycling is a special and highly sophisticated sport as racers should have various strategies for facing external forces and resistances to gain higher velocity. Cycling races include rode events, time (against the clock) event and criterioum (rotation) events. These different types of races include different velocities and race strategies. There is a motor equation that can be applied to calculate the most important external forces and variables affecting the ability of a cyclist in training and competition (3).

Gregor\&Conconi (2000) indicated that there are external factors that affect the mechanics of performance in cyclists. Racing on a level ground is totally different from racing on slops. There are special equations that indicate the difference in a cyclist's ability according to race velocity. Therefore, each bike is equipped with various gears and a cyclist should choose the best gear (front / rear) to use (gear ratio) (4).

Figure (1)

Shapes of front and rear gears and the gear shift

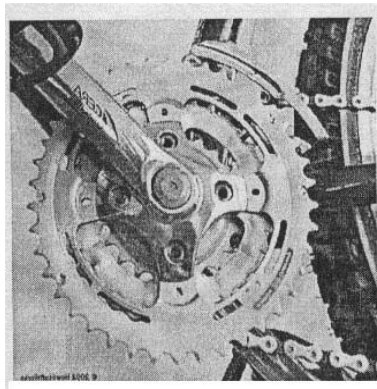

A

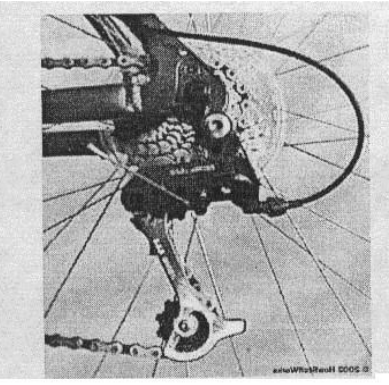

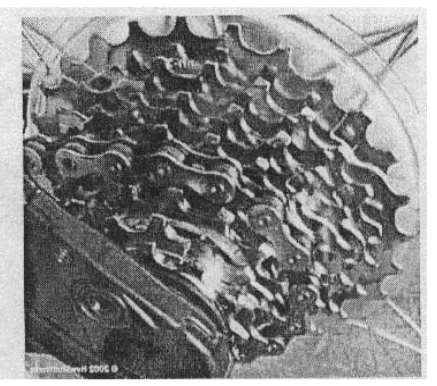

Motor equations are meant to identify a clear relation between the cyclist's ability and the bike velocity. Through calculating this result, we can identify the cyclist's efficiency.

Through these equations, we can make use of bike velocity as there are some factors affecting performance that are controllable, in addition to establishing strategies that differ according to the cyclist's level and expertise. Therefore, such variable should be measured for each individual cyclist so that we can tailor training accordingly.

The gear ratio equation, with reference to bike velocity and pedal rotations, is as follows:

$$
R=\frac{C_{\text {V-next }} \pi \times D_{\text {wheel }}}{\infty_{\text {comfort }}}
$$

As $\mathrm{CV}$ is the bike velocity $(\mathrm{m} / \mathrm{sec})$, Dwheel is the wheel diameter $(\mathrm{m})$ and $\infty$ is the ratio of pedal rotation per second (rps) (3).

Efficiency includes performance and the power needed to achieve it. The efficient skill is the skill that achieves a specific form due to a specific work exerted by muscles with the minimal energy cost. In other words, it means exerting maximal work with energy economics. It is important for athletes to achieve their motor goals through coping with performance problems that may contradict the energy saving principle (5).

In the light of basic goals of skills, which emerge from its mechanical goals, we can concentrate on developing the performance efficiency through training. The basic mechanical goal of cycling in general is to cover the farthest distance in the shortest duration (velocity). Previous knowledge of the goal develops the cyclist's ability to vary his/her performance at the same skill under various conditions through controlling the timing of motor series and organizing velocity (motion tempo and rhythm during race) through using only the suitable and needed muscles (6).

Cycling races are governed by general provisions indicated by the law. In other words, there is a motor task controlled by some conditions. To fulfill this task, the cyclist needs to perform a series of motions with specific time and place order. This is called "style". Each sports motion has it own criteria, duties and element that are well-known, either in the place 
of performance or in the bio-mechanical rules and principles of motion. When all these criteria and specifications are formed together, it leads to improving the motor skill level (6).

The researchers noticed that there is no stable mechanism for identifying the suitable gear for bike velocity according to scientific basics that enable coaches and cyclists to choose the suitable front and rear gears through the level, high and slope race paths. Therefore, the research problem is to identify an objective scientific mechanism for identifying the suitable gear for bike velocity. They also noticed that junior cyclists, more than more experienced ones, tend to reach higher velocities with the minimal number of rotations without knowing that this may lead to exertion and muscular stress more than working with the suitable gear and the suitable rate.

Due to scientific and technical developments in cycling and using modern technologies in training and equipments design/calibration, it is of major importance to cope with these gigantic developments in the field of cycling in Egypt.

\section{Aim}

The current research aims at designing and producing an electronic device that helps choosing suitable gear for the bike velocity according to pedal rotations of the cyclist. Therefore, cyclists can train according to correct and objective scientific bases.

\section{Methods}

\section{Pilot Study:}

The researchers measured the maximum rate of pedal rotations on each gear and with each velocity for a cyclist. Table (1) shows these results.

Table (1)

Measurements of maximum rate of pedal rotations/minute on each gear ratio with various velocities

\begin{tabular}{|c|c|c|c|c|c|}
\hline Velocity & \multirow{2}{*}{$30 \mathrm{~km}$} & \multirow{2}{*}{$35 \mathrm{~km}$} & \multirow{2}{*}{$40 \mathrm{~km}$} & \multirow{2}{*}{$45 \mathrm{~km}$} & \multirow{2}{*}{$50 \mathrm{~km}$} \\
\hline Gear & & & & & \\
\hline $52 / 17$ & 120 & 120 & 119 & 118 & 118 \\
\hline $25 / 16$ & 112 & 115 & 115 & 112 & 112 \\
\hline $52 / 15$ & 104 & 107 & 109 & 107 & 106 \\
\hline $52 / 14$ & 87 & 93 & 101 & 102 & 103 \\
\hline $52 / 13$ & 81 & 85 & 93 & 95 & 97 \\
\hline $52 / 12$ & 74 & 78 & 85 & 87 & 90 \\
\hline
\end{tabular}

Table (1) indicates the maximum rate of pedal 118RPM. For gear ratio 52/13 and a velocity of rotations on each gear and with each velocity for a cyclist. For gear ratio 52/17 and a velocity of $30 \mathrm{~km} / \mathrm{h}$, the pedal rotation rate was $120 \mathrm{RPM}$ $30 \mathrm{~km} / \mathrm{h}$, the pedal rotation rate was $80 \mathrm{RPM}$ while the same rate on a velocity of $45 \mathrm{~km} / \mathrm{h}$ was while the same rate on a velocity of $45 \mathrm{~km} / \mathrm{h}$ was 95RPM. 
Table (2)

Identifying gear ratio according to maximum Pedal Rotation per Minute for each player

\begin{tabular}{|c|c|c|c|c|c|c|c|}
\hline Gear ratio & Result & Velocity km/h & Velocity $\mathrm{m} / \mathrm{sec}$ & Rate & Near rate & Max rate $\mathrm{r} / \mathrm{min}$ & Load intensity \\
\hline $52 / 17$ & 3.058824 & 30 & 8.333333 & 76.52694 & 77 & 120 & 64.16667 \\
\hline $52 / 16$ & 3.25 & 30 & 8.333333 & 72.02535 & 72 & 112 & 64.28571 \\
\hline $52 / 15$ & 3.466667 & 30 & 8.333333 & 67.52377 & 68 & 104 & 65.38462 \\
\hline $52 / 14$ & 3.714286 & 30 & 8.333333 & 63.02218 & 63 & 87 & 72.41379 \\
\hline $52 / 13$ & 4 & 30 & 8.333333 & 58.5206 & 59 & 81 & 72.83951 \\
\hline $52 / 12$ & 4.333333 & 30 & 8.333333 & 54.01901 & 54 & 74 & 72.97297 \\
\hline $52 / 17$ & 3.058824 & 35 & 9.722222 & 89.28143 & 89 & 120 & 74.16667 \\
\hline $52 / 16$ & 3.25 & 35 & 9.722222 & 84.02958 & 84 & 115 & 73.04348 \\
\hline $52 / 15$ & 3.466667 & 35 & 9.722222 & 78.77773 & 79 & 107 & 73.83178 \\
\hline $52 / 14$ & 3.714286 & 35 & 9.722222 & 73.52588 & 74 & 93 & 79.56989 \\
\hline $52 / 13$ & 4 & 35 & 9.722222 & 68.27403 & 68 & 85 & 80 \\
\hline $52 / 12$ & 4.333333 & 35 & 9.722222 & 63.02218 & 63 & 78 & 80.76923 \\
\hline $52 / 17$ & 3.058824 & 40 & 11.11111 & 102.0359 & 102 & 119 & 85.71429 \\
\hline $52 / 16$ & 3.25 & 40 & 11.11111 & 96.0338 & 96 & 115 & 83.47826 \\
\hline $52 / 15$ & 3.466667 & 40 & 11.11111 & 90.03169 & 90 & 109 & 82.56881 \\
\hline $52 / 14$ & 3.714286 & 40 & 11.11111 & 84.02958 & 84 & 101 & 83.16832 \\
\hline $52 / 13$ & 4 & 40 & 11.11111 & 78.02747 & 78 & 93 & 83.87097 \\
\hline $52 / 12$ & 4.333333 & 40 & 11.11111 & 72.02535 & 72 & 85 & 84.70588 \\
\hline $52 / 17$ & 3.058824 & 45 & 12.5 & 114.7904 & 115 & 118 & 97.45763 \\
\hline $52 / 16$ & 3.25 & 45 & 12.5 & 108.038 & 108 & 112 & 96.42857 \\
\hline $52 / 15$ & 3.466667 & 45 & 12.5 & 101.2857 & 101 & 107 & 94.39252 \\
\hline $52 / 14$ & 3.714286 & 45 & 12.5 & 94.53328 & 95 & 102 & 93.13725 \\
\hline $52 / 13$ & 4 & 45 & 12.5 & 87.7809 & 88 & 95 & 92.63758 \\
\hline $52 / 12$ & 4.333333 & 45 & 12.5 & 81.02852 & 81 & 87 & 93.10345 \\
\hline $52 / 17$ & 3.058824 & 50 & 13.88889 & 127.5449 & 128 & 118 & 108.4746 \\
\hline $52 / 16$ & 3.25 & 50 & 13.88889 & 120.0423 & 120 & 112 & 107.1429 \\
\hline $52 / 15$ & 3.466667 & 50 & 13.88889 & 112.5396 & 113 & 106 & 106.6038 \\
\hline $52 / 14$ & 3.714286 & 50 & 13.88889 & 105.037 & 105 & 103 & 101.9417 \\
\hline $52 / 13$ & 4 & 50 & 13.88889 & 97.53433 & 98 & 97 & 101.0309 \\
\hline $52 / 12$ & 4.333333 & 50 & 13.88889 & 90.03169 & 90 & 90 & 100 \\
\hline
\end{tabular}

Table (2) shows how to identify the suitable gear according to the maximum rate of pedal rotation / minute for the cyclist. We can see that each velocity has a suitable gear so that the needed work does not exceed the necessary work for this velocity. Form table (2) we can see that the cyclist can get more rotations with gears of smaller momentum with a significant increase of velocity. Ali Abd El-Rahman and TalhaHosam El-Din (1981) indicated that each object revolves around an axis and this keeps it in rotation according to its angular motion unless it is affected by a de-central force that changes its condition (conclusion from Newton's first law. This is called "the principle of angular quantum continuity" (7)

Marlene (1995) indicated that increasing the mechanical power through increasing the exercise tempo (pedal rate) is better than increasing the gear volume. If the cyclist can not rotate the bigger gear, the mechanical power will decrease. Gear velocity and choosing the suitable gear reflect the needed power.
Choosing a bigger gear leads to the increase of muscular spasms and difficulty in rotating the pedal. Therefore, cyclists should be tested to identify their training level through rpm instead of excellence on the bigger gear (8)

\section{Research Community and Sample}

The researchers chose two cyclists from "ALGAISH" Sports Club, one for the pilot study and the other for verifying the device validity. The main sample subject was a member of the Egyptian national cycling team and one of the four high ranks in speed events.

\section{Research tools and equipment}

The researchers used some tools and equipment to design the device according to the following equation:

These tools and equipments are $R=\frac{C_{\text {V-next }} \pi \times D_{\text {wheel }}}{\infty_{\text {comfort }}}$ as follows:

1. A micro-controller (ATMEL 89 c52)

2. SERIAL EPROM 93 c85 memory

3. Crystal oscillator 7-15909 
4. Voltage regulator 7805

5. ACD 44780 screen

6. Transistor Bc557

7. Two magnetic sensors (send and receive)

8. Electric wire

9. Device software

10. Buzzer

11. Battery

During main experimentation, the researchers used the following:

1. A racing bike

2. Data collection form

3. A fixed home trainer bike

\section{Design phases:}

\section{First phase:}

The researchers studied the possibility to design and develop the device to verify its proto-type shape and how it works, compared to other similar products in the Egyptian and international markets. They also studied how to add it to the bike. This was done through investigating experts opinions in cycling, electric engineering and the head coach of the Egyptian cycling national team.

After that the researchers studied the equation carefully and verified all measurable variables. Then they bought the components that measure each variable carefully and chose the sensors suitable for the bike, referring to power and accuracy in getting results that will be transformed into numbers through the microcontroller to be displayed on screen.

Figure (2)

Front and back shots for the device at the first phase

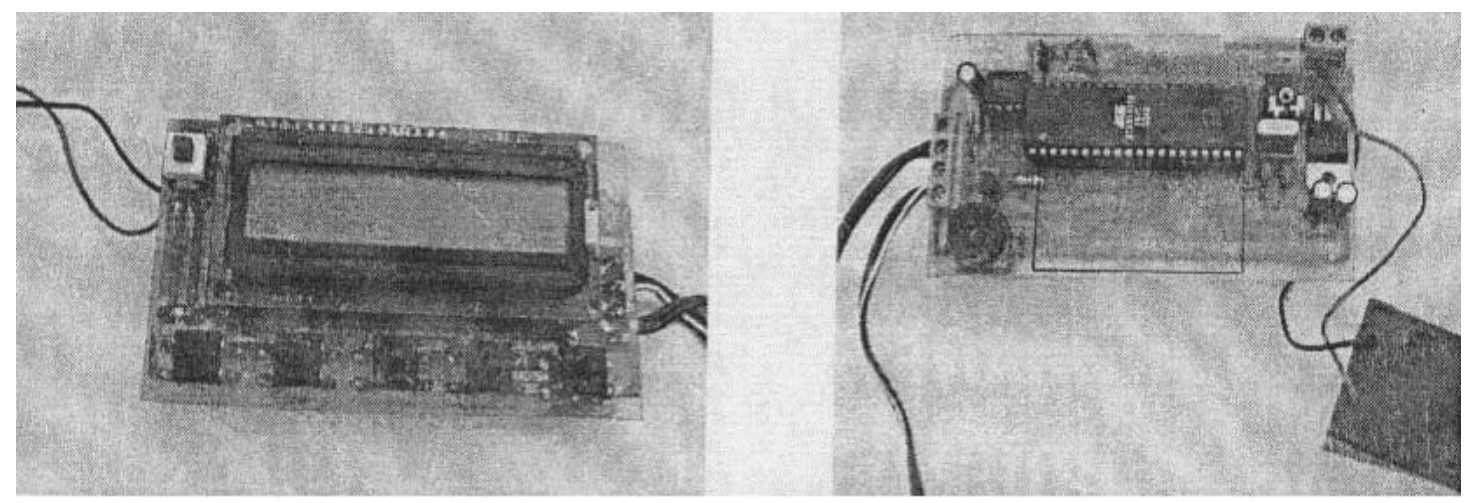

Figure (3)

Send and receive sensors (magnets)

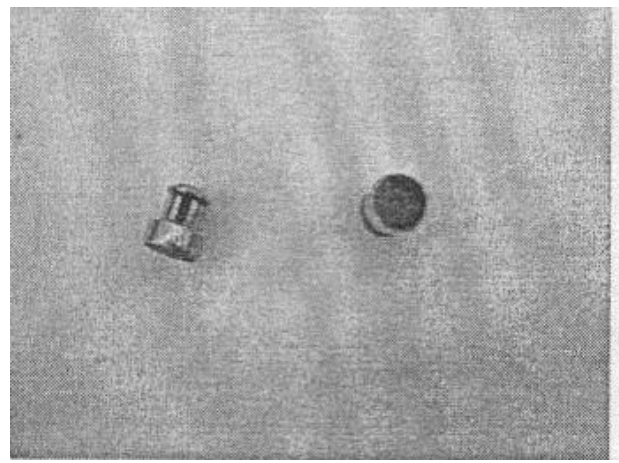

\section{Second phase:}

Each variable was measured independently to be displayed on screen (bike velocity, rotation rate ...etc). These measurements were linked to the equation and the micro-controller to be displayed. After that, screen settings were set (number of lines for digits display - data to be displayed clearly in front of the cyclist so that it

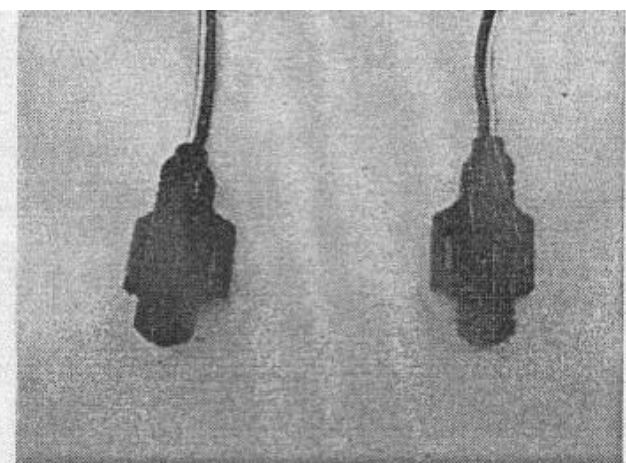

can include all the needed data without interrupting the cyclist's concentration on race).

\section{Third phase:}

In this phase, device parts were connected together in the electric lab (The Higher Institute of Technology - 10th of Ramadan City). Through trial, the researchers discovered some vibrations for the sensor as it has a magnetic field that affects screen readings. This was 
avoided by delaying the calculated time for calculating the number taken from the magnetic sensor and the micro-controller was programmed accordingly to avoid this interference.

Figure (4)

Rear shot for the device after connecting parts together.

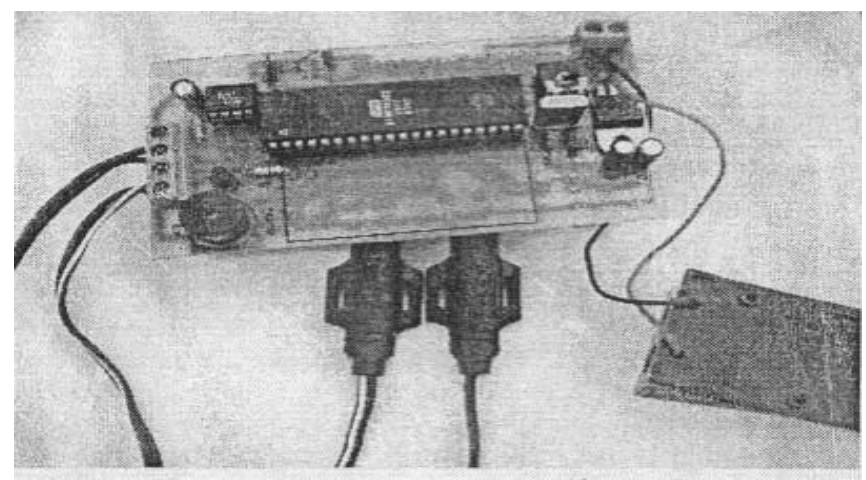

Figure (5)

Places of sensors on pedal and rear wheel.
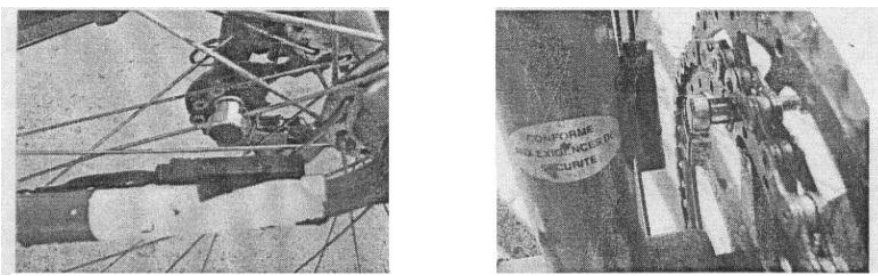

Figure (6)

Final shape of device.

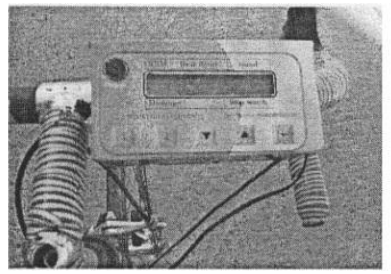

The main experiment:

The researchers performed the main experiment on the main sample member to measure the accuracy and suitability of the device, after modifications, then they used test/re-test technique (with 10-day time interval) to verify the device validity and reliability. Experts of cycling and electric engineering attended the main experiment in the athletic hall of The Higher Institute of Technology - 10th of Ramadan City.

The researcher recorded the measurement of the first and second experiments on the data collection form for the calculated data from the equation through assistants and results of screen reading to verify the device validity. First and second measurements were taken according to the same protocol to unify measurement conditions.

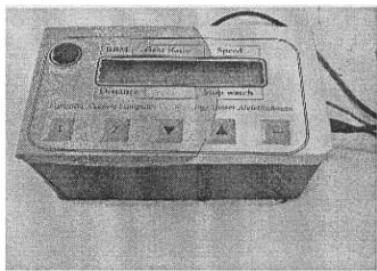

Figure (7)

Results displayed on screen

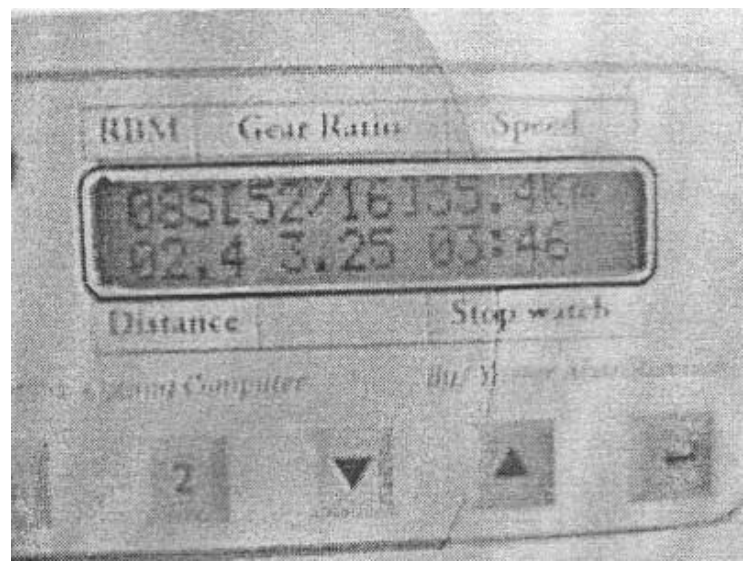

\section{Device Operation:}

1. Operating and calibrating device variables

2. Interring wheel diameter

3. Interring the big front gear (A) and small front gear (B)

4. Interring rear gears (8-10 gears) 
5. Interring front gears ratio to rear gears

6. Interring minimum and maximum velocity for each gear ratio to operate buzzer in case of any increase or decrease of the specified velocity for each gear according to the maximum pedal rotations

7. The cyclist is trained individually to follow the coach's instructions and instructions displayed on screen
8. The coach evaluates the cyclist's performance level formally

9. Asking the cyclist continuously about his feelings and evaluation of his own performance 10. Measuring heart rate continuously to followup the cyclist's effort and functional efficiency.

Figure (8)

Screen shots for device operation

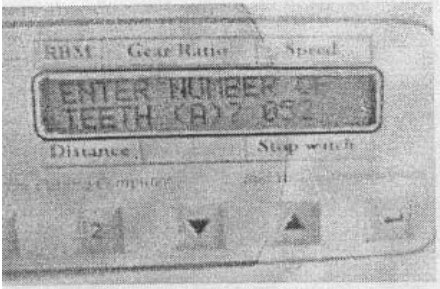

(3)

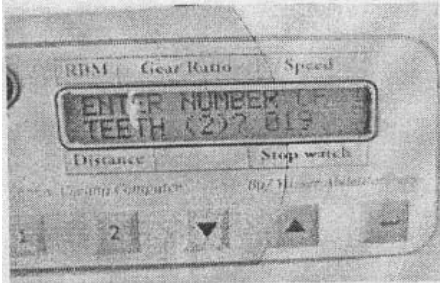

(6)

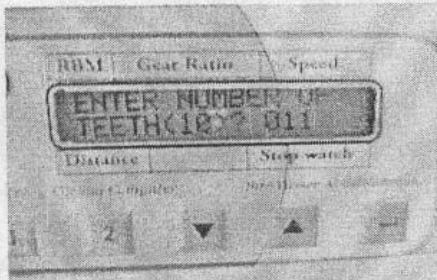

(9)

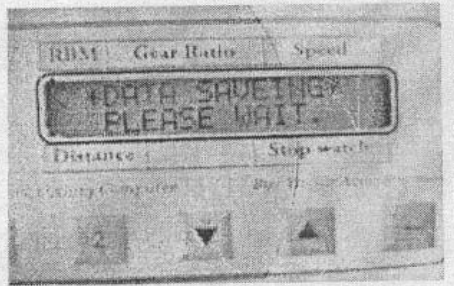

(12)

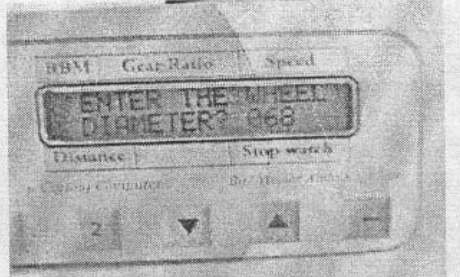

(2)

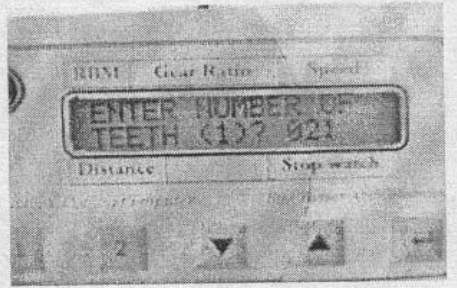

(5)

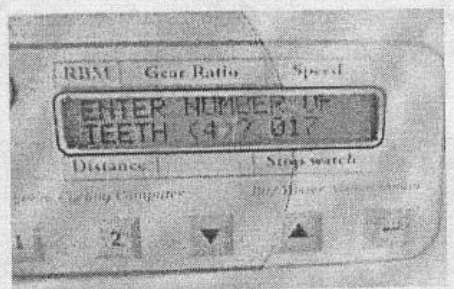

(8)

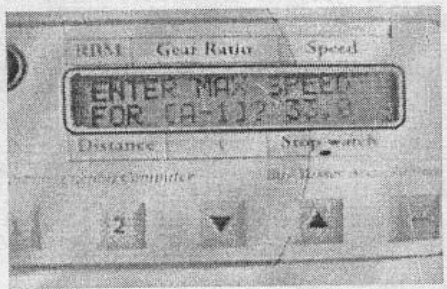

(11)

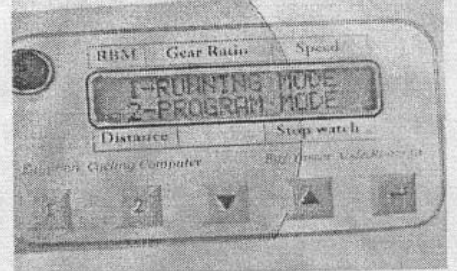

(1)

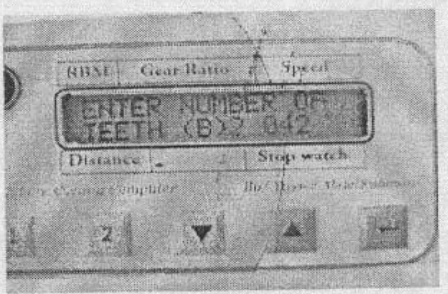

(4)

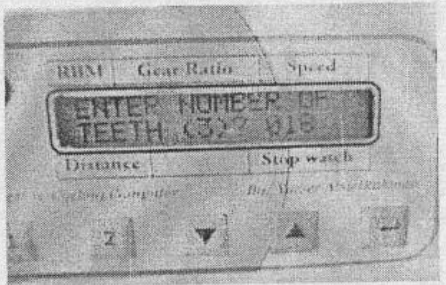

( 7 )

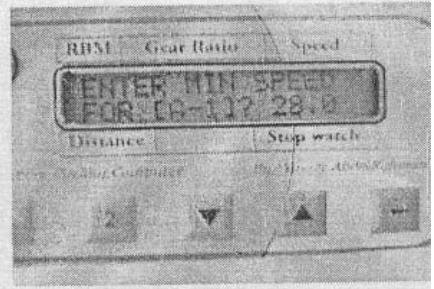

(10)

\section{Calibrating the device:}

The researchers tested the device several times during the main experimentation phase to verify its validity and any problems that may appear in design for each part when all parts are connected to work at the same time, and to identify any errors to be avoided during main execution. In addition, various tests may lead to better alternatives that can be invested in designing and constructing the device. This is accurately what happened as each test added more valuable ideas for modifying the device.

After designing and operating the device and verifying its validity for work, a technical committee of experts of electric engineering and sports training evaluated the technical aspects of 
the device and verified its validity as an effective equipment in cycling training in the athletic hall of The Higher Institute of Technology - 10th of Ramadan City.

According to the committee, validity of the device was $100 \%$. Validity through coefficients between data collected from device and data calculated through the equation was $100 \%$. Test/re-test reliability coefficient was $99 \%$. This indicates high rates of validity and reliability of device that reach $99 \%$.

Table (3)

Velocity and rotation rate values from device and from equation

\begin{tabular}{|c|c|c|c|c|c|c|c|c|}
\hline \multicolumn{5}{|c|}{ Device results } & \multicolumn{4}{|c|}{ Equation results } \\
\hline \multirow{2}{*}{ Gear ratio } & \multicolumn{2}{|c|}{ Rotation rate } & \multicolumn{2}{|c|}{ Velocity } & \multirow{2}{*}{ Gear } & \multirow{2}{*}{ Gear ratio } & \multirow{2}{*}{ Normal rotation by count } & \multirow{2}{*}{ Calculated velocity } \\
\hline & Mean & $\mathrm{SD} \pm$ & Mean & $\mathrm{SD} \pm$ & & & & \\
\hline 2.74 & 59 & 1.826 & 20.7 & 0.639 & $52 / 19$ & 2.736842 & 60 & 21.0452 \\
\hline 3.06 & 67.5 & 1.871 & 26.5 & 0.734 & $52 / 17$ & 3.058824 & 68 & 26.65726 \\
\hline 3.25 & 81.75 & 1.708 & 34.1 & 0.714 & $52 / 16$ & 3.25 & 82 & 34.15461 \\
\hline 3.47 & 90.6 & 0.894 & 40.3 & 0.394 & $52 / 15$ & 3.466667 & 91 & 40.43018 \\
\hline 3.71 & 104.8 & 1.924 & 49.9 & 0.917 & $52 / 14$ & 3.714286 & 105 & 49.98236 \\
\hline
\end{tabular}

Table (3) shows the mean and standard deviation values for rotation rate and velocity taken from device and from equation.

Table (4)

Device validity for measuring velocity and rotation rate

\begin{tabular}{|c|c|c|c|c|c|}
\hline \multirow{2}{*}{ Variable } & \multicolumn{2}{|c|}{ Device results } & \multicolumn{2}{|c|}{ Equation results } & \multirow{2}{*}{ Correlation coefficient } \\
\cline { 2 - 5 } & Mean & $\mathrm{SD} \pm$ & Mean & $\mathrm{SD} \pm$ & \\
\hline Velocity & 34.3 & 11.46 & 34.5 & 11.37 & 1.00 \\
\hline Rotation rate & 80.73 & 18.20 & 81.20 & 17.94 & 1.00 \\
\hline
\end{tabular}

Table (4) indicated that correlation coefficients were high and statistically significant (1.00) for both velocity and rotation rate. This indicates device validity.

Table (5)

Device test/re-test reliability for measuring velocity and rotation rate

\begin{tabular}{|c|c|c|c|c|c|}
\hline \multirow{2}{*}{ Variable } & \multicolumn{2}{|c|}{ Test } & \multicolumn{2}{c|}{ Re-test } & \multirow{2}{*}{ Correlation coefficient } \\
\cline { 2 - 5 } & Mean & SD \pm & Mean & SD \pm & \\
\hline Velocity & 34.3 & 11.46 & 34.62 & 11.17 & 0.999 \\
\hline Rotation rate & 80.73 & 18.20 & 81.65 & 17.36 & 0.999 \\
\hline
\end{tabular}

Table (5) indicated that correlation coefficients The researchers chose $25 \mathrm{~km}$ speed event were high and statistically significant (0.999) (individual) to show how the cyclist may cover for both velocity and rotation rate. This this distance depending on his own experience indicates device reliability.

\section{Final Phase:} in choosing gears and then another time after training on the device and getting alerts about suitable gear changes according to bike velocity

The purpose of this phase was to verify the device efficiency in training and competitions. and rotation rate as shown in table (6).

Table (6) pre- and post-measurements for the first technical test $(25 \mathrm{~km}$ speed event individual)

\begin{tabular}{|c|c|}
\hline Pre- measurement & Post-measurement \\
\hline 37.06 & 34.19 \\
\hline
\end{tabular}

Table (6) indicates that the performance level of the cyclist was improved by 2.47 minutes from pre- to post-measurement.

\section{Conclusions}

According to the research results and data analysis, the researchers concluded the following: 
1. The electronic device for identifying ideal gear ration, velocity, rotation rate, distance and time was valid.

2. The device software was valid according to the rules and regulation of the International Federation of Cycling.

3. The device software is adaptable according to variance in wheel diameter, number or rear gears and number of pins in each gear.

4. The device saves time and effort for coaches and cyclists.

5. The device works according the individual differences principle.

6. The device works according to logical and scientific bases.

7. The device helps coach to detect cyclists' errors during performance.

8. Compared to prices of other similar products, the device is low-cost. In addition, it can link all variables and analyze equations to calculate the ideal gear ratio.

\section{Recommendations}

According to research results and limitations, the researchers recommend the following:

1. Producing the device in cooperation with one of the leading sports equipments companies.

2. Generalizing the device in training for all cycling clubs and national teams to get better results.

3. It is important to establish specialized departments for sports engineering in the faculties of engineering and industrial higher institutes to integrate the expertise of specialists in sports and engineering for designing and developing scientific tools and equipments that fulfill the needs of sports in general, and especially bio-mechanics.

\section{References}

1. Gad, Mohamed A. (2001): Effects of gamelike exercises, using some technical equipments, on the digital level of front crawl junior swimmers. Master thesis, Faculty of Physical Education for Men - Helwan University Egypt. P:3. (in Arabic)

2. Thabet, Mostafa M.; Othman Mohamed S. \&Goda, Mostafa A. (1999): Sports engineering: theory and practice. The international scientific conference for physical education and sport between theory and practice. The scientific journal of physical education and sport, Faculty of Physical Education for Men - Helwan University - Egypt. PP:316-318. (in Arabic)

3. Swain, David P. (1998): Uphill and Downhill, Encyclopedia of sports medicine and science, $\mathrm{T}$. D. Fahey (editor). Internet society for sports science: http://www.sportsci.org

4. Gregor, Robert \&Conconi, Francesco (2000): Rode cycling, Blackwell science kk, mg, kodenmacho building, chuo-ku, Tokyo, Japan

5. Hosam El-Din, Talha H. (1993): BioMechanics: Theory and Practice. Dar Al-Fikr Al-Araby, Cairo - Egypt, P: 16 (in Arabic)

6. Al-Sabbagh, Nahed A. \& Aladdin, Gamal (1999): Kinesiology 7th ED. Faculty of Physical Education for men - Alexandria University Egypt. PP:42-44. (in Arabic)

7. Abd El-Rahman, Ali M. \&Hosam El-Din, Talha H. (1981): Sports Kinesiology and basics of motor analysis. Dar Al-Fikr Al-Araby, Cairo - Egypt, P: 256 (in Arabic)

8. Ordian, Marlene J. \& Cooper, John M. (1995): Biomechanics for human movement, WCB Brown, Bench Mark U.S.A

9. Huai, Ching\&ChingHuan (2003): An automatic transmission for bicycles: a simulation. www.elsevier.com/locate/ergon 